http://jmscr.igmpublication.org/home/ ISSN (e)-2347-176x ISSN (p) 2455-0450 crossref DOI: https://dx.doi.org/10.18535/jmscr/v9i3.38

\title{
Larvicidal Efficacy of Entomopathogenic Fungi Isolated from the Farmland Areas in Osogbo South-West Nigeria
}

\author{
Authors \\ Abideen Akinkunmi Wahab ${ }^{1}$, Busayo Janet Ayanwola ${ }^{2}$, Abolaji Tolulope Adeyemo ${ }^{2}$, \\ Ojevwe Harrison Egbo $^{3}$, Babatunde Adeola Afolabi ${ }^{4}$, Adeyemi Temitayo Adeyemo ${ }^{5^{*}}$ \\ ${ }^{1}$ Department of Microbiology, Osun State University, Osogbo, Nigeria \\ ${ }^{2}$ Department of Medical Microbiology and Parasitology, Ladoke Akintola University of Technology \\ Teaching Hospital, Osogbo, Nigeria \\ ${ }^{3}$ Department of Morbid Anatomy and Histopathology, Edo State University, Uzairue, Nigeria \\ ${ }^{4}$ Department of Family Medicine, Lautech Teaching Hospital, Osogbo, Osun State, Nigeria \\ ${ }^{5}$ Department of Medical Microbiology and Parasitology, Obafemi Awolowo University Teaching Hospitals \\ Complex, Ile-Ife, Nigeria \\ *Corresponding Author \\ Adeyemi Temitayo Adeyemo \\ Department of Medical Microbiology and Parasitology, Obafemi Awolowo University Teaching Hospitals \\ Complex, Ile-Ife, Nigeria
}

\begin{abstract}
Thousands of entomopathogenic fungi are in existence, unexploited and it is important to identify and use for biocontrol of mosquito larva. The aim of this study was to determine the larvicidal efficacy of newly identified entomopathogenic fungi. Soil samples were taken from three different locations in a farmland at Osun State University (UNIOSUN),Osogbo, Nigeria for isolation and identification of entomopathogenic fungi and test its larvicidal effect on mosquito larvae. The samples were analyzed using spread plate technique which include serial dilution of samples, inoculation on prepared Saboraud Dextrose Agar using three dilutions $\left(10^{-}, 10^{-}{ }^{3}, 10^{-10}\right)$ and incubation at $27^{0} \mathrm{c}$ for 72 hours after which the plates were examined for growth followed by sub-culturing. Mosquito larvae were breeded, picked into sterile distilled water in Petri dishes. The spores of the fungi were scraped on the larva to determine the larvicidal effect of the fungal isolates at varied interval: 40 minutes, 2 hours, 24hours, and 48hours. A total of seven fungi were isolated two (Aspegillus flavus and Aspergillus niger) of which were able to show larvicidal effect on mosquito larvae. Some larvae died at exactly 40 minutes of inoculation when tested with Aspergillus niger, some took hours (48hours) while others remain viable. This study concludes that Aspergillus niger and Aspergillus flavus isolated from soil samples exhibited biolarvicidal efficacy against mosquito larvae and the mortality rate increases with time of exposure. Entomopathogenic fungi should therefore be explored for control of mosquitoes in our environment.
\end{abstract}

\section{Introduction}

Mosquitoes are well known vector of human and animal pathogens and millions of people are killed by mosquito-borne diseases every year such as malaria dengue, chikungunya, Zuka, yellow fever, encephalitis and filariasis. ${ }^{1}$ Vector control, 
sanitation, habitat disruption or personal protection from mosquito bites are the most widely measures employed to control and protect people from infection of these diseases. ${ }^{2}$ Over the past few decades, many countries organized official programs of mosquito vector control.

Currently, synthetic chemical pesticides against adults or larva have been the mainstay and are the most widely used for control of Mosquitoes. Mosquito larvae are the attractive targets for these pesticides because mosquitoes breed in water and thus, it is easy to deal with them in this habitat ${ }^{3}$ The indiscriminate use of chemical insecticides to target adult mosquitoes has given rise to problems such as mosquito resistance, environmental contamination, and health risk to humans and nontarget organisms. To mitigate these problems, there is an urgent need to develop alternatives to conventional chemical insecticides, which are safe, effective, biodegradable and highly selective. There has been an increasing awareness in the possibility of using biological control agents as alternative to chemical control of mosquitoes. Among the eminent biological control agents are entomopathogenic microorganisms such as fungi as larvicides. ${ }^{4}$ Fungal biocontrol agents are the most important among all the Entomopathogenic microorganisms due to easy delivery, chances to improve formulation, vast number of pathogenic strains known, easy engineering techniques and its ability to control both sap sucking pests such as mosquito and aphids as well as pest with chewing mouth parts. They include numerous phylogenetically. morphologically and ecologically diverse fungal species which evolve to exploit insects with their main route of entry being through the insect's integument, by ingestion or via wounds or trachea. ${ }^{5}$ Most entomopathogenic fungi can be grown on artificial media (shin et al., 2009); being natural mortality agents which are environmentally safe, there is a worldwide interest in the use and manipulation of entomopathogenic fungi for biological control of insects and other arthropod pests. ${ }^{6}$ They display a higher degree of effectiveness in infecting their host, acting as regulators for numerous harmful insects including both domestic and forest insects. ${ }^{7}$

In general mosquitoes show susceptibility towards entomopathogenic fungi and its derived products. They have low toxicity to non-target organisms and using entomopathogenic fungi as larvicides may be a promising approach for biological control of mosquitoes due to their selective toxicity and ready decomposability in the ecosystem. ${ }^{8}$ Also, unlike the inherent dangers which are associated with the process of production of synthetic insecticides, the process for the manufacture of microbial products is safe and less pollutant. ${ }^{9}$

This study therefore aimed at determining the larvicidal activities of fungal isolates with a view to exploring their potential for biological and environmental control of mosquito-borne diseases.

\section{Materials and Methods \\ Collection of Soil Samples}

The study area was a farmland within Osun State University, Osogbo, Nigeria. About 100g of soil samples from $10-15 \mathrm{~cm}$ deep were aseptically collected with sterilized hand trowel into sterile Aluminum foil paper from three different locations on the same farmland and transported to the laboratory for further analysis.

\section{Isolation of Fungi in the Soil Samples}

Fungi were isolated from soil samples using serial dilution method; one gram (1g) of soil sample was suspended in $9 \mathrm{ml}$ of sterile diluents and serially diluted up to tenth factor $\left(10^{-10}\right)$. Dilutions of $10^{1}$, $10^{5}$ and $10^{10}$ was used to isolate the fungi. With sterile syringe, $0.1 \mathrm{ml}$ of each dilution was drawn and inoculated into the plates which have been labeled and arranged according to dilution, then glass spreader dipped into $100 \%$ ethanol and flamed was used to spread the inoculums in the petri dish by slightly opened beside the flame to avoid contamination. All plates were incubated at room temperature $\left(25^{\circ} \mathrm{C}-27^{\circ} \mathrm{C}\right)$ and observed for growth between 3 to 5 days i.e. (72 hours -120 hours). The process was carried out in duplicate. 


\section{Sub culturing of Fungal Isolates}

Distinct fungal colonies were aseptically picked from the mixed culture using sterile inoculating needle and transferred onto a freshly prepared sterile SDA plates and incubated for $3-5$ days at $25^{\circ} \mathrm{C}$. This process was repeated until pure cultures were obtained pure culture was maintained on SDA slant and stored at $4{ }^{\circ} \mathrm{C}$ in the refrigerator for further analysis.

\section{Macroscopic and Microscopic Identification of Isolated Fungi}

Each pure culture plate was observed for morphology characteristics of the isolates including color, growth rate and texture.

For presumptive identification of isolated fungi from soil samples, a drop of lactophenol blue was placed on a clean glass slide, and an inoculating needle was used to pick from the pure culture plate onto the stain on glass slide. The cover slip was gently placed on the prepared smear, mounted and view under X40 objective on Microscope. The Microscopic appearance was captured and compared with the structures in soil fungi Compendium. ${ }^{10}$

\section{Breeding of Mosquito Larvae}

Buckets containing half-filled dechlorinated water were placed under shade at different site locations for 7 days around the school General Laboratory of the institution. After breeding, the buckets were transported into the laboratory.

\section{Rejuvenating of the Isolates}

Each fungal isolate was taken from the stock culture and inoculated on freshly prepared sterilized Saboraud Dextrose agar plates, incubated at room temperature $\left(25^{\circ}-27^{\circ} \mathrm{C}\right)$ for 72 hours.

\section{Procedure of Biolarvicidal Efficacy}

One milliliter of sterile distilled water was dispensed into each petri dish onto which the spores of fungal spores were scraped and the concentration standardized and determined by measuring its optical density (turbidity) (labeled as higher concentration) by previously documented protocols, ${ }^{11,12}$ then four larvae were picked and transferred into the spore suspension in the petri dishes. A lower spore concentration was prepared by further diluting $1 \mathrm{ml}$ of the standard concentration (prepared as above) with $1 \mathrm{ml}$ of distilled water to decrease the concentration to half (labeled as lower concentration), the same quantity of larvae (four larvae) were then introduced into this spore suspension. Control treatment contains sterile distilled water and mosquito larvae in a Petri dish. Death or viability of mosquito larvae was monitored and recorded at room temperature $\left(25^{\circ} \mathrm{C}-27^{\circ} \mathrm{C}\right)$ from the point of inoculation to three days.

\section{Confirmation Test}

To confirm that the fungal isolates were responsible for the mortality of mosquito larvae, the dead larvae were picked and inoculated on freshly prepared Saboraud Dextrose Agar. The growth of the fungal isolates was shown on the plates after $72 \mathrm{hrs}$.

\section{Results}

\section{Fungi species Isolated from Soil Samples}

A total of seven (7) fungi were isolated from the soil samples and they included Microsporum spp., Fusarium spp., Malbranchea entero arthric, Aspergillus niger, Aspergillus flavus, Onychocola Canadensis, and Arthrinium spp.

Table 1 and Figure $1 \mathrm{a}$ and $1 \mathrm{~b}$ show the morphological characteristics and microscopic appearances of the isolates through direct observation of colonies on solid media plates as well as viewing under $\mathrm{x} 40$ objectives.

\section{Result of Biolavicidal Effect of Fungi}

Biolarvicidal potential of isolated fungi was carried out and it revealed that Aspergillus niger, Aspergillus flavus have efficacy against the mosquito larvae, but the rate at which death occur in each isolate is different. Result is shown in Table 2 and 3.

\section{Confirmation of Biolavicidal Effect of Fungi}

This shows that the fungi isolates were responsible for the mortality of mosquito larvae 
by observing growth of fungi on solid media plates after incubation.

\section{Mortality Rate of Mosquito Larvae after Treatment with Fungi}

Table 2 shows that isolate A-1a (Microsporum spp.) had no larvicidal effect on the all the four larvae treated at lower spore concentration $(25.25 \mathrm{mg} / \mathrm{L})$, the same effect was observed for isolates A-1b (Fusarium spp.), B-1c (Malbranchea entero arthric), C-5a (Onychocola canadensis) and C-5c (Arthrinium spp.) at the same spore concentration.

However, at the same spore concentration, isolate B-5c (Aspergillus niger) had no effect at 2hours on the four larvae but some effect at 24hours in which two larvae were viable while two were dead (50\% mortality rate). At 48 hours all the four larvae were dead (100\% mortality rate). Likewise, isolate B-5d (Aspergillus flavus) shows no mortality at 2 hours but some effect at 24hours in which two larvae were viable while two were dead (50\% mortality rate). At 48 hours all the four larvae were dead (100\% mortality rate).

Table 3 shows mortality rates at a higher spore concentration $(50.5 \mathrm{mg} / \mathrm{L})$. Aspergillus niger showed $100 \%$ mortality at different time of exposure ranging from 40 minutes to 24 hours. This implies that Aspergillus niger has high larvicidal potency regardless of time of exposure. On the other hand, at the same spore concentration, Aspergillus flavus showed no larvicidal effect on the mosquito larvae at 40 minutes, 50\% mortality at 2hours and $100 \%$ mortality at 24 hours implying that the mortality rate with Aspergillus flavus increases with time of exposure as represented in Figure

Table 1: Cultural morphology and characteristics of the isolates

\begin{tabular}{lccc}
\hline SAMPLE & SURFACE/REVERSE & GROWTH RATE & TEXTURE \\
CODE & White wooly surface/rusty brown at the reverse & Grow slowly & Velvety to wooly \\
\hline A-1a & Whitish and brown at the reverse & Rapid growth & Wooly to cottony \\
A-1b & White wooly & Moderately wooly & Wooly \\
B-1c & Rlack surface, yellowish filament and reverse & Rapid growth & Powdery \\
B-5c & White becoming yellowish/pale & Rapid growth & Powdery and wooly \\
B-5d & Green with whitish wooly growth/yellowish at the base & Moderately rapid & Smooth to slightly \\
C-5a & White wooly surface and brown at the reverse & Moderate growth & Wooly to cottony \\
C-5c & & &
\end{tabular}

Isolate Surface picture code

A-1a

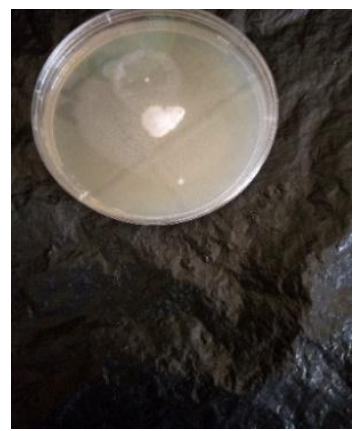

Reverse picture

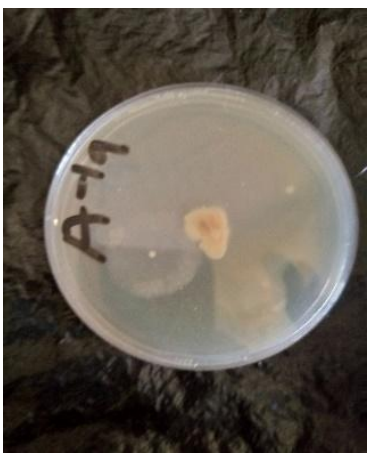

Microscopic view

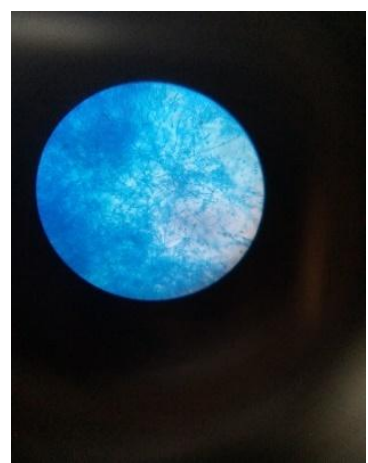

Presumptive identification

Microsporum spp. 


\section{JMSCR Vol||09||Issue||03||Page 224-232||March}

A-1b

B-1c
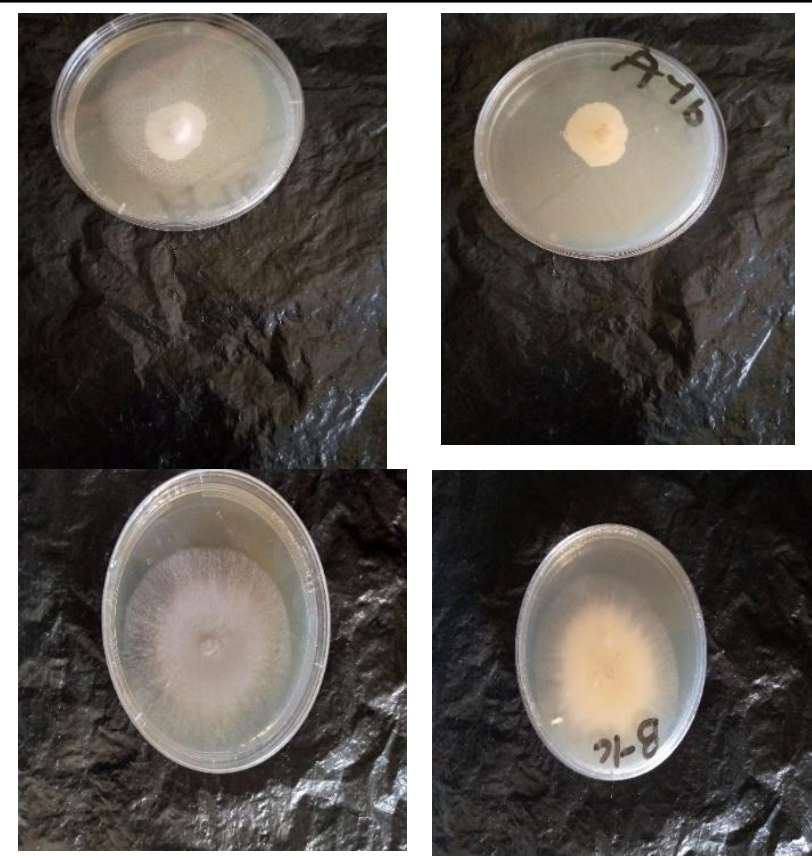

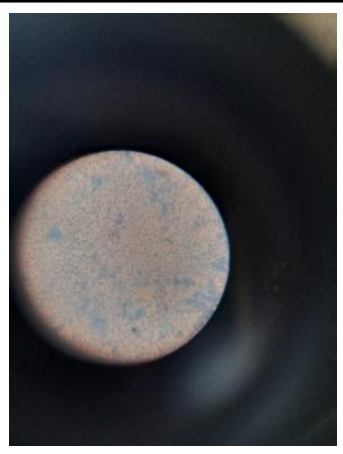

Fusarium spp.

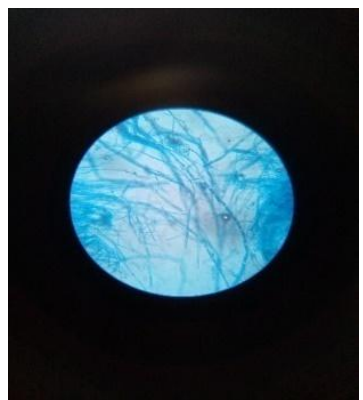

Malbranchea spp.

Figure 1a: Macroscopic and microscopic appearances of the isolates

B-5c
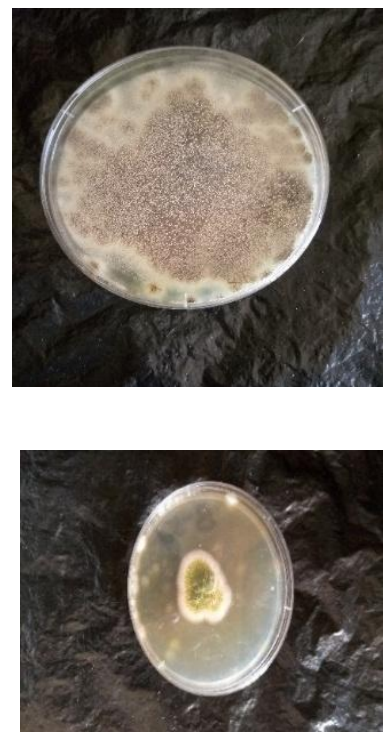

C-5a

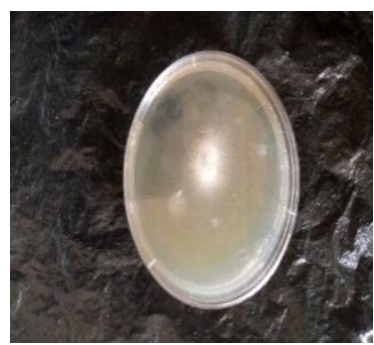

C-5c

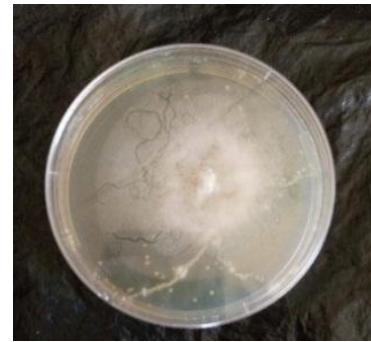

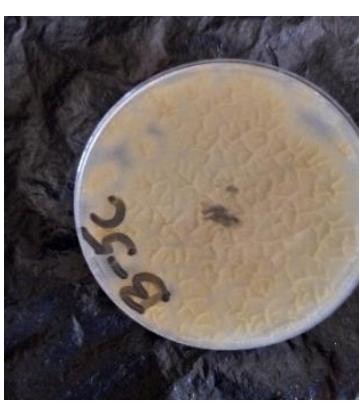
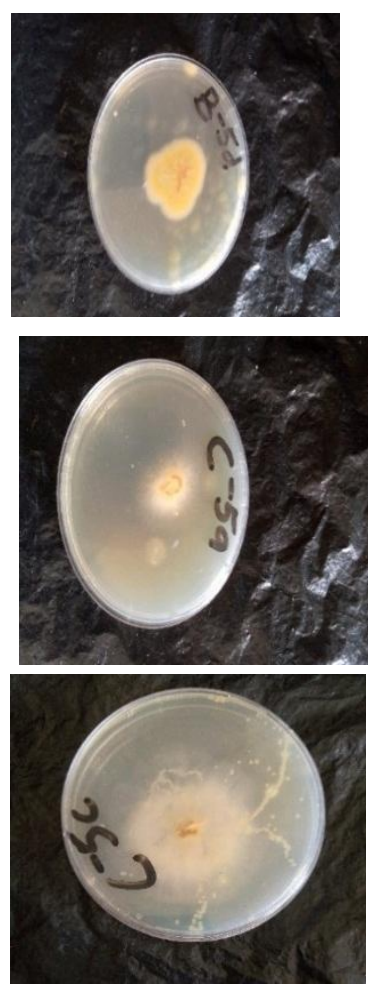

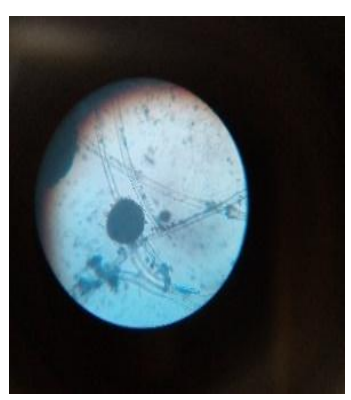

Aspergillus niger
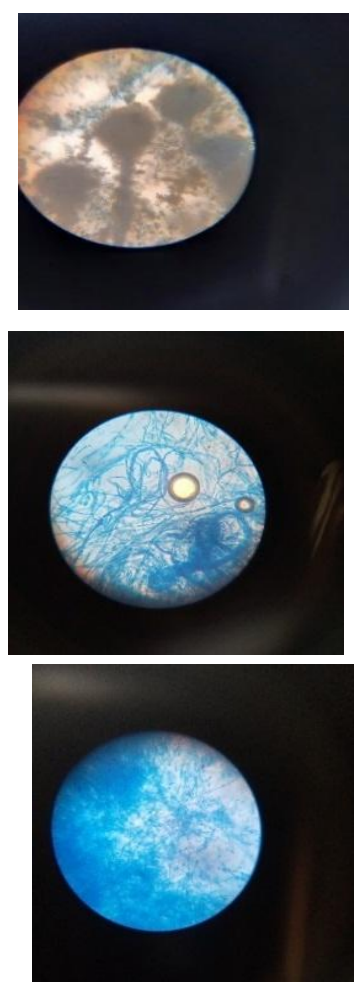

Aspergillus flavus

Onychocola

canadensis

Arthrinium spp.

Figure 1b: Macroscopic and microscopic appearances of the isolates 


\section{JMSCR Vol||09||Issue||03||Page 224-232||March}

Table 2: Biolarvicidal efficacy of lower concentration of fungi isolates on Mosquito larvae

\begin{tabular}{lll}
\hline ISOLATE CODE & NUMBER OF LARVAE IN 2MLS OF & PERIOD OF INNOCULATION \\
& LOWER CONCENTRATION FUNGAL &
\end{tabular}
LOWER CONCENTRATION FUNGAL SUSPENSION

\begin{tabular}{llccc}
\hline & & 2 hrs. & 24 hrs. & 48 hrs. \\
A- 1a & 4 & $4 \mathrm{~V}$ & $4 \mathrm{~V}$ & $4 \mathrm{~V}$ \\
A-1b & 4 & $4 \mathrm{~V}$ & $4 \mathrm{~V}$ & $4 \mathrm{~V}$ \\
B-1c & 4 & $4 \mathrm{~V}$ & $4 \mathrm{~V}$ & $4 \mathrm{~V}$ \\
B-5c & 4 & $4 \mathrm{~V}$ & $2 \mathrm{D} 2 \mathrm{~V}$ & $4 \mathrm{D}$ \\
B-5d & 4 & $4 \mathrm{~V}$ & $2 \mathrm{D} 2 \mathrm{~V}$ & $4 \mathrm{D}$ \\
C-5a & 4 & $4 \mathrm{~V}$ & $4 \mathrm{~V}$ & $4 \mathrm{~V}$ \\
C-5c & 4 & $4 \mathrm{~V}$ & $4 \mathrm{~V}$ & $4 \mathrm{~V}$ \\
\hline
\end{tabular}

$\mathrm{V}=$ Viable

$\mathrm{D}=$ Dead

Table 3 Biolarvicidal efficacy of higher concentration of fungi Isolates on Mosquito larvae

\begin{tabular}{lcccc}
\hline ISOLATE CODE & $\begin{array}{c}\text { NUMBER OF LARVAE IN } \\
\text { 2MLS OF HIGHER } \\
\text { CONCENTRATION OF } \\
\text { FUNGAL SUSPENSION }\end{array}$ & & & \\
\hline A-1a & 4 & 40 minutes & $2 \mathrm{hrs}$ & $24 \mathrm{hrs}$ \\
A-1b & 4 & $4 \mathrm{~V}$ & $4 \mathrm{~V}$ & $4 \mathrm{~V}$ \\
B-1c & 4 & $4 \mathrm{~V}$ & $4 \mathrm{~V}$ & $4 \mathrm{~V}$ \\
B-5c & 4 & $4 \mathrm{~V}$ & $4 \mathrm{~V}$ & $4 \mathrm{~V}$ \\
B-5d & 4 & $4 \mathrm{D}$ & $4 \mathrm{D}$ & $4 \mathrm{D}$ \\
C-5a & 4 & $4 \mathrm{~V}$ & $2 \mathrm{D} 2 \mathrm{~V}$ & $4 \mathrm{D}$ \\
C-5c & 4 & $4 \mathrm{~V}$ & $4 \mathrm{~V}$ & $\mathrm{~V}$ \\
\hline V- & $4 \mathrm{~V}$ & $\mathrm{~V}$ & \\
\hline
\end{tabular}

$\mathrm{V}=$ Viable

$\mathrm{D}=$ Dead

Sample Code

$\mathrm{B}-5 \mathrm{c}$

$B-5 d$
Name of the Isolates

Aspergillus niger

Aspergillus flavus
Picture of the Isolates
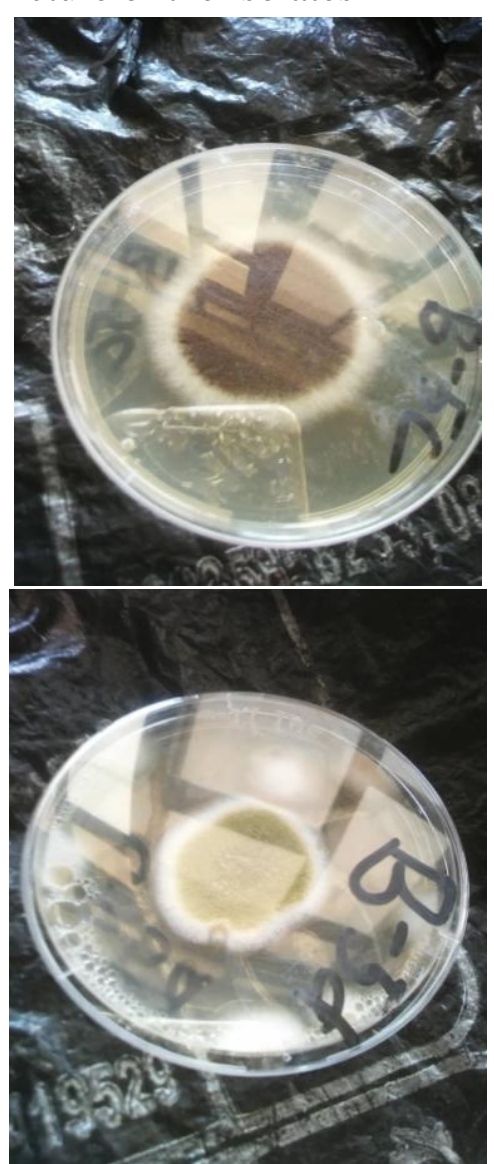

Figure 2: Confirmation test result showing growth of mosquito larvae on solid media 


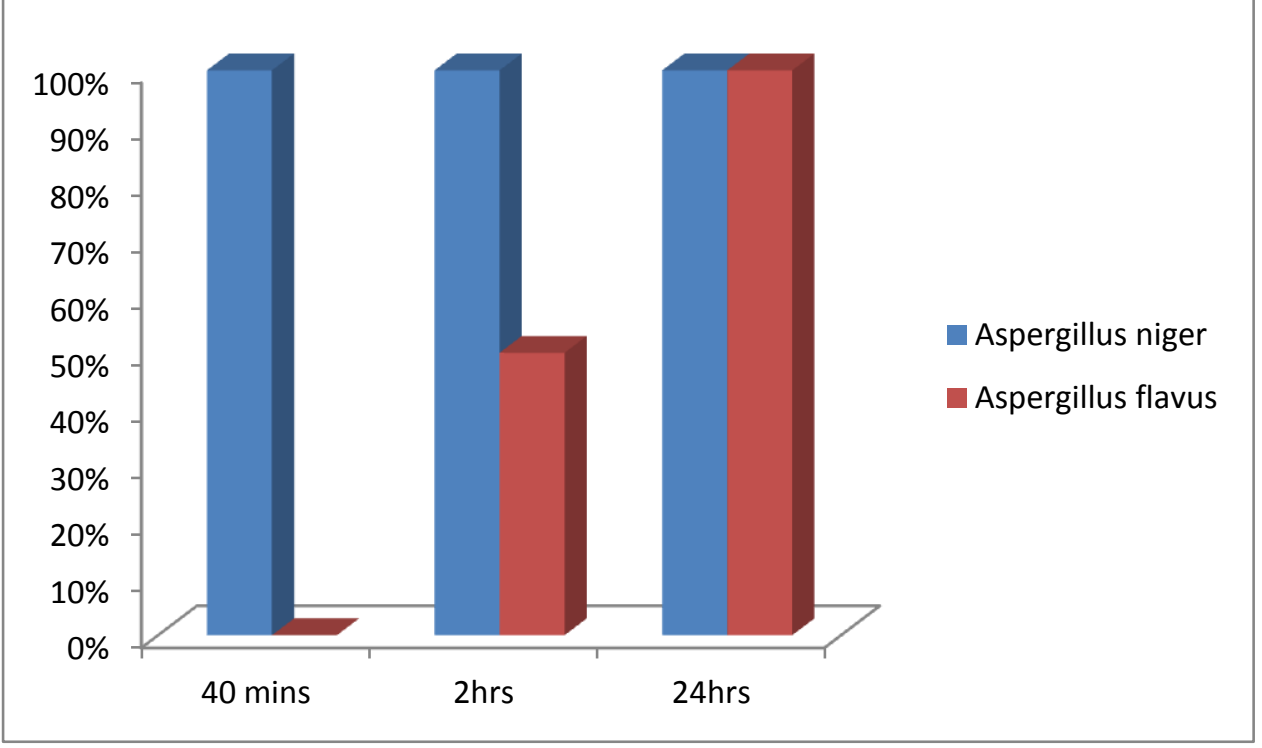

Figure 3: A chart showing the mortality rate of mosquito larvae at a concentration of 2 larvae per milliliter (4 per 2 millilitres) at different durations

\section{Discussion}

The researches on the larvicidal potential of microbes particularly fungi for controlling the mosquito larvae have only been extensively carried out on adult mosquito for several decades. Entomogenous fungi have caused high mortalities in mosquito population in many laboratories including Aedes and Anopheles species. ${ }^{13}$ However, a review of studies by elucidates the larvicidal potentials of fungi and revealed that the fungi could successfully infect and kill larvae of a wide range of mosquito genera with varying rates of mortality. ${ }^{6}$ Furthermore, a research conducted in East Africa to determine the pathogenicity of entomopathogenic fungi against several strains of adult Anopheles gambiae revealed a high infection rates ranging from 46 to $88 \%$ with Metarhizium anisopliae being the most pathogenic strain. ${ }^{14}$ In recent years, there is a considerable amount of attention focusing on identifying potential mosquitocidal fungus from natural sources for effective control of mosquitoes as a key measure to curtailing the vector borne diseases in human. A study done in Asia researching the larvicidal potential of Lagenidium giganteum, a water weed, noted its efficacies in killing the tested vectors with appreciable safety to non-target organisms and good biological stability. ${ }^{15}$ According to a large-scale field trial conducted in the United States, mycelium of Lagenidium giganteum caused 40-90\% infection rates in Culextarsalis and Anopheles freeborn sentinel larvae. ${ }^{16}$

The potentials of many fungi have been established for mosquito control, nevertheless, only a few have received commercial attention and are marketed for use in vector control programmes globally. It is therefore becoming obvious that entomopathogenic fungi promises a greater roles in expansion of the limited options available for effective mosquito control, thereby contributing in a substantial and sustainable manner to prevention and control of vector-borne diseases.

In this study, seven fungi were isolated from soil samples and been identified as: Microsporum spp., Fusarium spp., Malbranchea entero arthric, Aspergillus niger, Aspergillus flavus, Onychocola canadensis, Arthrinium spp. The larvicidal potential of these fungi were tested at different concentration against mosquito larvae at different period of exposure (40 minutes, 2hours, 24hours and 48hours). Two isolates which were Aspergillus niger, and Aspergillus flavus exhibited larvicidal potential against mosquito larvae while other five isolates showed no effect on the larvae. At a lower fungal spore 
concentration $(25.25 \mathrm{mg} / \mathrm{L})$, Aspergillus niger showed no mortality at 2hours, $50 \%$ mortality at 24 hours and $100 \%$ mortality at 48 hours. At the same spore concentration, Aspergillus flavus showed similar mortality rates at the same time of exposure as Aspergillus niger. At higher spore concentration $(50.5 \mathrm{mg} / \mathrm{L})$, Aspergillus niger showed a mortality rate of $100 \%$ mortality at 40 minutes, 2hours and 48hours. This is similar to the study by Mauryaet al. who evaluated larvicidal potential of Aspergillus niger, Aspergillu sflavus, Aspergillus parasiticus against Anopheles stephensi and Culex quinque fascistus with Aspergillus flavus showing greatest bio efficacy at $50 \%$ lethal concentration (LC50) against larvae of Anopheles stephensi and Culex quinquefasciatus at 24 hours of exposure and bio efficacy increased in both larval species at 48 hours. ${ }^{17}$

At the same higher concentration, Aspergillus flavus showed no mortality at 40 minutes, 50\% mortality rate at 2 hours and $100 \%$ mortality rates at 24 hours which also similar to the research of Govindraja et al who evaluated larvicidal effect of Aspergillus flavus and other fungi against Culex quinque fascistus with high mortality rate. ${ }^{18}$ Similarly, a study conducted in northern Nigerian showed that the fungal concentration required to kill $50 \%$ of larvae decreased as the exposure duration (in hours) increased. It also reported that larval mortality rates increased proportionately with concentration of pathogenic fungi. ${ }^{8}$ According to a study by Bukari et al. fungal spores of entomopathogenic fungi, especially when formulated in synthetic oil as an effective carrier, provided a better lavicidal effect with significant reduction of vector by up to $50 \%$ in Kenya, emphasizing its promising role for controlling larval populations of mosquitoes. ${ }^{9}$

The usefulness of fungal biolavicidal effects for potentiating the synthetic (chemical) lavicides, even at low (environment-friendly) concentrations, had recently been studied. An Indian study demonstrated the synergistic potential of Aspergillus flavus and Temephos (an organophosphate lavicide) by showing dramatic reduction in malaria transmission due to enhanced larval mortality and promoted good larval control at more moderate levels of coverage even in areas where insecticide resistance makes control ineffective. ${ }^{4}$

\section{Conclusion and Recommendations}

From the result of this study, Aspergillus niger and Aspergillus flavus isolated from soil samples exhibited biolarvicidal efficacy against mosquito larvae. It was also shown that the mortality rate increases with time of exposure. These isolates are therefore potential be deployed for mosquito control programs singularly or in combination with other control agents. Further studies on larvicidal activity of fungi species should be carried out in this environment for evidence-based information to further establish the usefulness of these species as myco-insecticides to combat mosquito and other arthropods which are causative agents of human diseases.

Acknowledgements; The authors are grateful to the technical staff of Department of Microbiology, Osun State University, Osogbo, Nigeria.

Competing interests; The authors declare that they have no competing interests.

Authors' contributions; BJA and AAW conceived and designed the study. BJA and AAW conducted laboratory experiments. ATA, OHE, BAO and ATA wrote the initial draft, ATA, OHE, BAO and ATA edited and reviewed the final manuscript. All authors approved the final report.

Funding: This study was self-sponsored.

Availability of data and material: The datasets used and analysed during the current study are available from the corresponding author on reasonable request.

Disclaimer: The views expressed in the submitted manuscript are authors and not an official position of the institution.

\section{References}

1. Odalo J,Omolo M, Malebo H, Angira J, Njeru $\mathrm{P}$, Ndiege I. Repellance of essential oil of 
some plants from Kenya Coast against Anopheles gambiae. Acta Tropica 2005; 95: 210-18.

2. World Health Organization (WHO), (2013). Global Programme to eliminate lymphatic filariasis. (Available on www.who.int).

3. Arivoli S., Ravindran J.K., Tennyson S., (2012). Larvicidal Efficacy of plant Extracts against the malaria vector Anopheles stephensis Liston (Diptera:Culicidae).World J. MedSci 7:77-80.

4. Bhan S, Shrankhla, Mohan L, Srivasstava CN. Larvicidal toxicity of Temephos and entomopathogenic fungus, Aspergillus flavusand their synergistic activity against malaria vector, Anopheles stephensi. Journal of Entomology and Zoology Studies 2013; 1 (6): 55-60.

5. Watarabe T. Pictorial Atlas of soil and seed fungi morphologies of cultured fungi 2010; P295-296.

6. Scolte EJ, Knols BGJK, Samson RA,Takken W. Entomopathogenic fungi for Mosquito control: A review.Journal Insect Science 2004; 4:19.

7. Augustyniuk-Kram A, Kram K J, Lo JA. Entomopathogenic Fungi as an Important Natural Regulator of Insect Outbreaks in Forests (Review). Forest ecosystem- more than Just Trees 2012; P265-94. (Available on www.intechopen.com).

8. Soni N, Soam P. Effect of Chrysosporium keratinoplulum metabolites against Culex quinquefasciantus after chromatographic purification. Parasitol Res 2010; 107: 132936 .

9. Bukhari T, Willem T. Development of Metarhizium anisopliae and Beauveria bassiana formulations for control of malaria Mosquito larvae. Parasites \& Vectors 2011; 4(1):23. DOI: 10.1186/1756-3305-4-23.

10. Ellis D, Davis S, Alexiou H, Handke R, Bartley R. Descriptions of medical fungi. 2nd ed. Adelaide, South Australia; 2007.
11. Morris SC, Nicholls PJ. An evaluation of optical density to estimate fungal spore concentrations in water suspensions. Phytopathology 1978; 68: 1240-42.

12. World Health Organization. (2005). Guidelines for laboratory and field testing of mosquito larvicides. World Health Organization.

https://apps.who.int/iris/handle/10665/69101

13. Kerwin JL, Washino RK. Field evaluation of Lagenidium giganteum (Oomycetes :Lagendales) and description of a natural epizootic involving a new isolates of fungus. J. Med. Entomol 1988; 25: 452-60.

14. Scolte EJ, Takken W, Knols BGJ. Pathogenicity of five East Africa entomopathogenic fungi to Adult Anopheles gambiae (Diptera: culicidae). Proc. Exper. Appl. Entomol. Nev Amsterdam 2003; 1425 9.

15. Vyas N, Dua KK, Prakash S. Efficacy of Lagenidium giganteum metabolites on mosquito larvae with reference to non-target organism. Parasitol. Res 2007; 101: 385-90.

16. Kerwin JL, Washino RK. Ground and aerial application of the asexual stage of Coelomomyces giganteum for control ofmosquitoes associated with rice culture in the central valley of California. Journal of the American Mosquito Control Association 1987; 3: 59-64.

17. Maurya P, Mohan L, Sharma P, Srivastava $\mathrm{CN}$. Evaluation of larvicidal potential of certain insect pathogenic fungi extracts against Anopheles stephensi and Culex quinquefasciatus. Entomological Research 2011; 41(5): 211-15.

18. Govindraja M, Jebamesan A, Reetha D. Larvicidal effect of extracellular secondary metabolites of different fungi against the mosquito, Culex quinquesfasciatus. Tropical Biomedicine 2005; 22: 1-3. 